\title{
Utilização de Algoritmos Genéticos no Processo de Formação de Equipes Heterogêneas em Turmas Universitárias
}

\author{
Yuri Kelvin N. Silva ${ }^{1}$, Fabrício V. A. Guerra ${ }^{2}$, Flavius L. Gorgônio ${ }^{2}$ \\ ${ }^{1}$ Departamento de Informática e Matemática Aplicada (DIMAp) \\ Universidade Federal do Rio Grande do Norte (UFRN) \\ Av. Sen. Salgado Filho, 300 - 59.078-970 - Natal - RN - Brasil \\ ${ }^{2}$ Departamento de Computação e Tecnologia (DCT) \\ Universidade Federal do Rio Grande do Norte (UFRN) \\ Rua Joaquim Gregório, 296 - 59.300-000 - Caicó - RN - Brasil \\ \{yuriikeelvin, fabriciolo\}@gmail.com, flavius@dct.ufrn.br
}

\begin{abstract}
The allocation of students into teams is one of the strategies used in Education in order to favor collaborative learning through the exchange of knowledge between individuals. However, the proccess of assigning students to heterogeneous teams is still a problem related to this approach, given the number of possible combinations. This work proposes a solution based on genetic algorithms to optimize the process of formation of heterogeneous teams in a university environment. The carried out experiments demonstrated that the proposed strategy was able to maximize the heterogeneity in teams more effectively than traditional methods commonly used for this activity.
\end{abstract}

Resumo. A alocação de alunos em equipes é uma das formas utilizadas na Educação para favorecer a aprendizagem colaborativa através da troca de conhecimentos entre indivíduos. Entretanto, realizar a distribuição dos alunos em equipes heterogêneas ainda é um problema relacionado a essa abordagem, dada a quantidade de combinações possíveis. Este trabalho propõe uma solução baseada em algoritmos genéticos para otimizar o processo de formação de equipes heterogêneas em ambiente universitário. Os experimentos realizados demonstram que a estratégia proposta foi capaz de maximizar a heterogeneidade nas equipes de forma mais eficaz que os métodos tradicionais comumente utilizados para essa atividade.

\section{Introdução}

Nos dias atuais, é imprescindível a utilização de equipes para a realização e solução de tarefas, atividades e problemas. Nenhum indivíduo é capaz de atuar como uma unidade isolada, de ter acesso a todas as informações, bem como, possuir tempo e habilidades cognitivas necessárias para realizar tarefas mais complexas [Cooke et al. 2013]. Apesar do ambiente ser o mesmo para todos os membros de uma equipe, suas perspectivas são heterogêneas, isto é, cada indivíduo possui conhecimentos e habilidades distintos, tornando impossível para qualquer um assumir todas as perspectivas e possuir consciência completa da tarefa ou situação.

Nesse contexto, o aprendizado de forma colaborativa, a partir da interação dos alunos em equipes heterogêneas, é visto como um processo de construção do conhecimento 
VIII Congresso Brasileiro de Informática na Educação (CBIE 2019)

Anais do XXX Simpósio Brasileiro de Informática na Educação (SBIE 2019)

decorrente da participação, do envolvimento e da contribuição ativa dos alunos na aprendizagem uns dos outros [Torres and Amaral 2011]. Segundo [Maldonado Pérez 2007], o emprego da aprendizagem colaborativa no âmbito acadêmico facilita o intercâmbio de conhecimentos e habilidades entre os alunos, permitindo o conflito de diferentes pontos de vista e opiniões e auxiliando na revalorização das perspectivas próprias.

Metodologias tradicionalmente utilizadas para distribuição de alunos em equipes baseiam-se principalmente em duas abordagens: i) distribuição randômica, conduzida de forma aleatória pelo professor; e ii) distribuição por afinidade, conduzida normalmente pelos próprios alunos que escolhem seus pares para compor as equipes. Na primeira abordagem, dado o fator aleatoriedade e o grande número de possíveis combinações, não há nenhuma garantia de que as equipes formadas sejam igualmente heterogêneas. $\mathrm{Na}$ segunda abordagem há um complicador adicional, uma vez que a maioria dos alunos escolhe seus pares baseados nas similaridades entre si, prejudicando ainda mais a heterogeneidade.

Apesar dos benefícios inerentes à aprendizagem colaborativa em equipes heterogêneas, a complexidade em distribuir manualmente os alunos em equipes ideais (ou próximas ao ideal, maximizando-se as diferenças) torna-se um impedimento à utilização dessa estratégia, dado o grande número de possibilidades de combinar os alunos em diferentes equipes, que cresce de forma exponencial à medida em que aumenta o número de alunos nas turmas [Silva et al. 2012]. Todavia, algoritmos aproximativos apresentam-se como uma abordagem comumente utilizada na solução tarefas complexas e humanamente árduas, minimizando a vasta quantidade de combinações em meio aos estados possíveis no espaço de busca e sendo capazes de obter, em tempo hábil, uma solução aceitável ao problema [Blum and Roli 2003].

A partir dessas considerações, este artigo endereça o seguinte problema de pesquisa: é possível otimizar o processo de formação de equipes através do uso de algoritmos evolucionários, de modo a potencializar a heterogeneidade entre os integrantes de cada equipe? Nesse sentido, propõe-se a aplicação de um algoritmo genético para otimizar o processo de formação de equipes heterogêneas em turmas universitárias. O algoritmo agrupa e realoca os indivíduos entre equipes através de uma métrica de mensuração da heterogeneidade que baseia-se no desempenho acadêmico. O objetivo é maximizar a heterogeneidade de conhecimentos entre os indivíduos da equipe. A proposta é validada a partir da comparação dos resultados com outros métodos tradicionais de formação de equipes, como a escolha aleatória e a escolha por afinidades (de acordo com as preferências dos próprios alunos).

As seções seguintes deste artigo estruturam-se como segue: a seção 2 expõe uma revisão da literatura de algoritmos genéticos para a formação de equipes; a seção 3 descreve a solução proposta frente ao problema da formação de equipes heterogêneas; os experimentos efetuados e a validação da proposta estão discutidos na seção 4; por fim, as considerações finais e trabalhos futuros estão presentes na seção 5.

\section{Revisão da Literatura}

Os algoritmos genéticos (AGs) são uma técnica potencial de otimização aplicada nas mais diversas áreas do conhecimento humano, inclusive na Educação. Nessa perspectiva, [Moreno et al. 2012] propuseram um método baseado em uma abordagem de AG para ob- 
VIII Congresso Brasileiro de Informática na Educação (CBIE 2019)

Anais do XXX Simpósio Brasileiro de Informática na Educação (SBIE 2019)

ter equipes inter-homogêneas e intra-heterogêneas. O método permite a consideração de diversas características estudantis e é validado por meio da comparação com a estratégia de agrupamento aleatório e agrupamento auto-organizado.

Em [Chen and Yang 2014], os autores utilizaram dois métodos para agrupar alunos em favor da aprendizagem colaborativa. Primeiramente, um algoritmo de agrupamento foi aplicado sobre um conjunto de dados dos alunos objetivando dividi-los em diferentes equipes com características semelhantes. Em seguida, um AG foi aplicado para dividir as equipes de acordo com os conhecimentos dos alunos. Os resultados foram propícios ao objetivo proposto, alcançando a divisão automática das equipes e encontrando, de forma aproximada, as equipes de aprendizagem colaborativa, validando a proposta por meio de simulações.

[Cen et al. 2015] utilizaram um AG para maximizar o desempenho médio esperado do trabalho em equipe ou a melhoria média alcançada por um aluno como resultado da aprendizagem em equipe. A abordagem proposta baseia-se na otimização global para identificar a ótima alocação dos alunos nas equipes a fim de satisfazer um dos critérios. Os resultados não só ajudaram a compreender os fatores significativos que influenciam o desempenho de equipes em aprendizagem colaborativa como também forneceram estratégias práticas sobre a composição ideal das equipes para atividades em aprendizagem colaborativa.

[Lin et al. 2016] aplicaram um AG, em conjunto com uma técnica de preferência, para facilitar a otimização dos agrupamentos para um sistema de suporte a grupos baseado na web, a fim de ajudar os educadores na formação de equipes inter-homogêneas e intraheterogêneas. Os experimentos mostraram que a proposta possui resultados relevantes em relação ao método aleatório e ao método utilizado em [Moreno et al. 2012].

Em [Zheng et al. 2016], uma abordagem híbrida baseada em AGs e otimização por enxame de partículas foi utilizada para a formação de equipes heterogêneas na aprendizagem colaborativa com base nas características dos alunos. Para validar o método proposto, uma série de experimentos foram realizados a partir de oito conjuntos de dados com diferentes níveis de complexidade. A abordagem proposta foi comparada com o método exaustivo e o método randômico, obtendo melhor desempenho e alcançando soluções de alta qualidade em um tempo de execução aceitável.

Em um trabalho recente desenvolvido por [Balieiro et al. 2017], os autores apresentaram uma abordagem baseada em AG para formação de grupos de estudos em ambientes virtuais de aprendizagem. $\mathrm{O}$ esquema proposto considerou informações pertinentes aos alunos para a realização dos agrupamentos. Apesar de objetivarem a homogeneidade, os resultados foram significativos em relação ao método aleatório.

Sendo assim, diante dos trabalhos apresentados, verifica-se que os AGs têm sido adotados como uma técnica potencial para o problema de formação de equipes. O presente trabalho distingue-se dos demais por seguir o contexto de pesquisa descrito em [Silva et al. 2012], uma abordagem que não utiliza AGs, mas que considera exclusivamente o fator heterogêneo nas equipes, visto que essa característica tende a agrupar os alunos de modo a contribuir significativamente para a aprendizagem por meio da troca de conhecimentos distintos, além de ter um direcionamento prático para vivências reais no âmbito do ensino superior. 
VIII Congresso Brasileiro de Informática na Educação (CBIE 2019)

Anais do XXX Simpósio Brasileiro de Informática na Educação (SBIE 2019)

\section{Solução Proposta}

A solução proposta para otimizar o processo de formação de equipes heterogêneas tem por fundamento os AGs, que podem ser compreendidos como uma técnica computacional inspirada no processo da evolução biológica por meio da sobrevivência dos indivíduos mais adaptados, sendo descoberto como um método aproximativo útil para a otimização de problemas [Holland 1992]. A ideia básica dos AGs é encontrar a solução ótima (ou uma solução quase ótima) em um conjunto de soluções candidatas, utilizando dos operadores genéticos de seleção, recombinação e mutação nas populações, avaliando cada nova população (cromossomos) gerada de modo que tais soluções estejam convergindo para o objetivo desejado. Assim, a ideia da solução proposta, em si, é a de implementar um AG para a formação de equipes heterogêneas, testando a heterogeneidade das equipes por ele formadas em contraposição à heterogeneidade de equipes formadas a partir de técnicas tradicionalmente adotadas em sala de aula.

Perante esses aspectos, o AG proposto objetiva alocar alunos com diferentes perfis acadêmicos em um mesmo agrupamento, favorecendo a heterogeneidade (Figura 1). Inicialmente, um conjunto de soluções viáveis ao problema é obtido através permutações aleatórias de alunos da turma sobre a qual serão efetuados os agrupamentos, caracterizando assim os cromossomos iniciais do AG. Ao longo das gerações, os cromossomos são avaliados e submetidos à seleção, recombinação e mutação. A expectativa é de que, ao final do processo, um cromossomo possua uma distribuição de alunos em equipes que maximiza a heterogeneidade entre eles. Vale salientar que, por tratar-se de um método de solução aproximada e, possivelmente, o número de alunos de cada perfil não ser o mesmo, a configuração das equipes propostas pelo AG pode conter indivíduos de perfis semelhantes em um mesmo agrupamento, ainda que a heterogeneidade seja garantida.

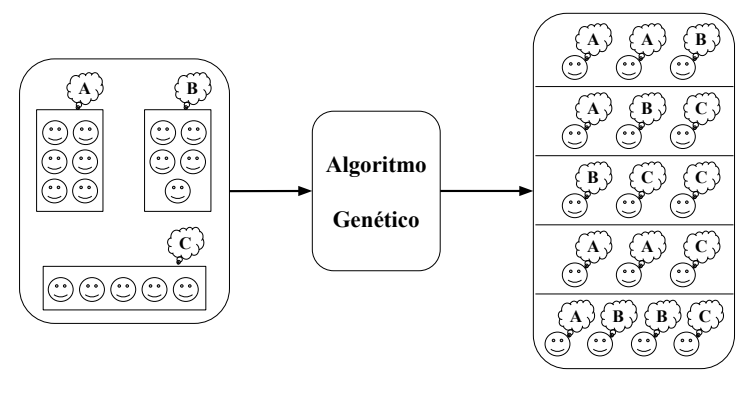

Figura 1. llustração da proposta do trabalho

\subsection{Estrutura e Caracterização do Cromossomo}

A representação cromossomial adotada neste trabalho baseia-se em um vetor de números inteiros que retratam o índice de cada aluno no conjunto original de dados (Figura 2). Cada gene $x$ pertencente a um cromossomo $y$ refere-se ao índice do vetor de notas do respectivo aluno $x$. Cada vetor de notas possui um tamanho $p$ que corresponde à quantidade de disciplinas. O tamanho dos cromossomos varia de acordo com a quantidade $n$ de alunos alocados em uma turma.

\subsection{Operadores Genéticos}

O processo de execução do AG fundamenta-se na progressiva utilização dos operadores de seleção, cruzamento e mutação em prol da melhoria das soluções. A etapa de seleção 
VIII Congresso Brasileiro de Informática na Educação (CBIE 2019)

Anais do XXX Simpósio Brasileiro de Informática na Educação (SBIE 2019)

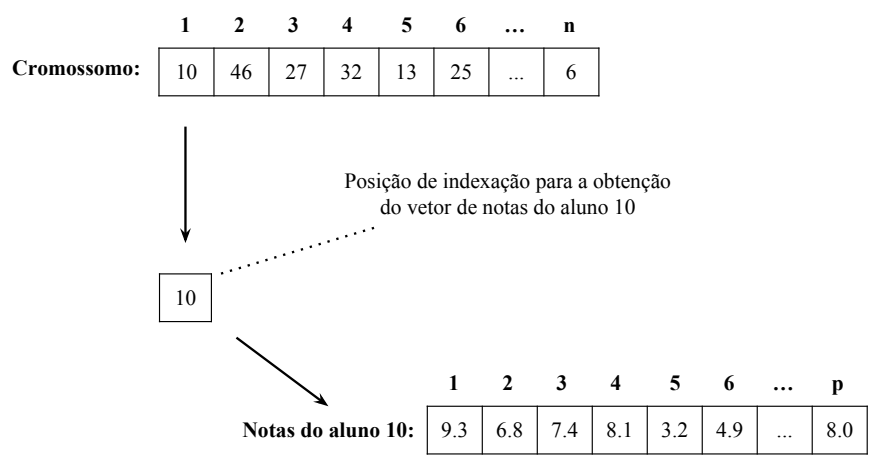

Figura 2. Estrutura do cromossomo

é responsável pela escolha dos pares de cromossomos que irão ser recombinados a fim de gerar os filhos da nova geração. Para tal, utilizou-se o método da roleta, sendo esta uma técnica usualmente empregada nos AGs [Coley 1999]. O método consiste em fornecer probabilidades de seleção com base no fitness do cromossomo. Assim, cromossomos com melhor valor de fitness tendem a ser selecionados com maior frequência, aumentando as chances de transferência dos melhores genes e aprimoramento do código genético nas próximas gerações.

A etapa seguinte à seleção é dada pelo cruzamento dos cromossomos pais que foram selecionados na etapa anterior. Com o propósito de preservar boas distribuições de alunos em equipes, um operador de cruzamento é proposto (Figura 3). A partir dos cromossomos pais (Figura 3a), o operador atua selecionando $r$ equipes do pai $1, r<n$ onde $n$ é o número total de equipes, para compor as equipes do filho, mantendo-as nas respectivas posições do cromossomo (Figura 3b). As equipes remanescentes são escolhidas a partir do pai 2. Para os casos em que há repetição de indivíduos na estrutura do cromossomo do filho, tais indivíduos são substituídos aleatoriamente por outros que não estejam, obrigatoriamente, na composição das equipes advindas do pai 1.

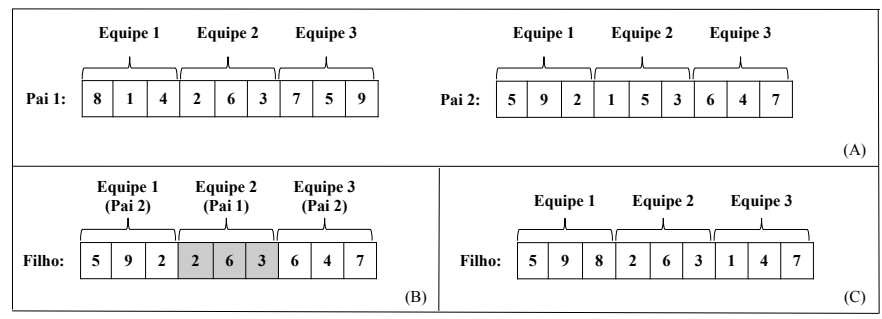

Figura 3. Exemplo do operador de cruzamento

[Mitchell 1998] destaca que o cruzamento é o principal mecanismo de inovação e variação nos AGs, entretanto, apenas sua utilização poderá convergir os pontos para soluções situadas em máximos locais. Para isso, o operador de mutação atua como deslocador dos pontos no espaço de maneira que as buscas sejam realizadas em outros locais. No esquema proposto (Figura 4), dois indivíduos são selecionados aleatoriamente desde que pertençam a equipes distintas (Figura 4a). Em seguida, uma permutação é realizada (Figura 4b) de modo que novas composições de equipes sejam obtidas, tornando possível atingir configurações de equipes que maximizem a heterogeneidade. 
VIII Congresso Brasileiro de Informática na Educação (CBIE 2019)

Anais do XXX Simpósio Brasileiro de Informática na Educação (SBIE 2019)

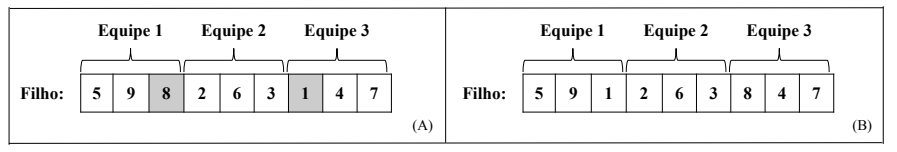

Figura 4. Exemplo do operador de mutação

\subsection{Função de Avaliação}

Nos AGs, a determinação da qualidade de uma solução é efetuada pela função de avaliação. Uma vez que o problema é definido, a função de avaliação será responsável por avaliar e indicar o quão adequada está a solução ao problema. Para o AG proposto, a função de avaliação é dada por um índice de validação para mensurar a dissimilaridade entre os indivíduos de um agrupamento. Assim como em [Silva et al. 2012], adotou-se o índice Davies-Bouldin (DB) por sua viabilidade, simplicidade e eficácia ao cálculo da heterogeneidade dos agrupamentos. Uma explicação mais detalhada acerca do procedimento de cálculo do índice DB pode ser encontrada em [Bandyopadhyay and Maulik 2002].

Isto posto, o processo de aplicação do DB é descrito a seguir. Cada cromossomo possui uma divisão de equipes de uma turma tal que cada equipe é composta por $k$ alunos. Para os casos em que a quantidade de alunos na turma não admite uma divisão exata de equipes de tamanho $k$, os alunos remanescentes são distribuídos aleatoriamente nas equipes já constituídas. O fator que define a dissimilaridade entre os alunos no cálculo do DB é o desempenho acadêmico nos componentes curriculares obrigatórios cursados, sendo esta uma das formas mais comuns de agrupar alunos em equipes [Macintyre and Ireson 2002]. Portanto, o objetivo do AG proposto foi maximizar o resultado do índice DB, tal que este representasse o maior valor de heterogeneidade obtido em uma solução. A Figura 5 apresenta um exemplo do cálculo do DB para uma distribuição de equipes.

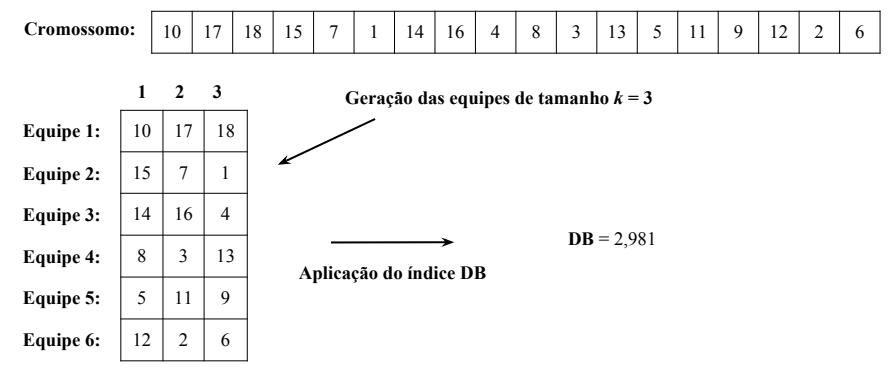

Figura 5. llustração da avaliação de um cromossomo

\section{Metodologia}

A metodologia adotada neste trabalho foi validar a solução proposta através de um experimento científico que permitisse a comparação do desempenho (em termos de heterogeneidade) do $\mathrm{AG}$ proposto em face às propostas tradicionais. A variável-dependente do experimento foi o índice $\mathrm{DB}$, medido sobre as equipes formadas, como expressão de sua heterogeneidade. A ideia foi observar, inicialmente, se o AG apresentava resultados positivos em relação às demais propostas sobre as amostras utilizadas nos experimentos para, 
em caso afirmativo, verificar, através de testes estatísticos inferenciais, se esses resultados positivos observados se sustentavam para além das amostras.

O experimento para a avaliação da proposta teve como amostra dados de estudantes do curso de Bacharelado em Sistemas de Informação da Universidade Federal do Rio Grande do Norte. Os dados coletados correspondem às notas dos estudantes e foram obtidos a partir de uma base de dados envolvendo um total de 7 turmas (de 2009 a 2015), 35 componentes curriculares (disciplinas) e 128 estudantes.

\subsection{Pré-processamento dos Dados}

A etapa de pré-processamento foi necessária para o tratamento de valores faltosos nos dados coletados. Para os casos de reaproveitamento de disciplinas em outras instituições, os dados foram preenchidos por meio do cálculo do valor médio das notas dos demais alunos, sendo esta uma prática empregada e aceita para tal circunstância [Larose 2014]. Para os casos em que o aluno realizou o trancamento ou cancelamento da disciplina, ou sequer cursou a mesma, atribui-se o valor 0 para a nota do respectivo componente curricular. Esta métrica visa o preenchimento dos valores faltosos sem que afetem nos resultados dos experimentos.

\subsection{Parâmetros de Configuração do Algoritmo}

Durante o processo de execução dos experimentos, alguns parâmetros de configuração do AG foram fixados para a obtenção dos resultados. A justificativa para a escolha desta condição decorre devido ao escopo do trabalho limitar-se em uma análise comparativa de métodos de agrupamentos para a formação de equipes. Logo, apesar da natureza dos AGs fundamentar-se na combinação de diferentes fatores para buscar uma melhor configuração das soluções, não coube a este trabalho analisar quais parâmetros desempenhariam melhores resultados para tal ocasião. Tendo em vista esses aspectos, os parâmetros definidos para o AG estão presentes na Tabela 1.

[Mitchell 1998] apresenta que estes valores definidos para o número de gerações, o tamanho da população e as taxas de cruzamento e mutação são costumeiramente adotados para problemas que envolvem AGs. O número estipulado para a quantidade de alunos por equipe é um valor médio comumente utilizado pelos docentes para a formação de equipes nas turmas da instituição considerada. É importante destacar que os número de gerações do AG foi adotado também como a quantidade de iterações da escolha aleatória.

Tabela 1. Parâmetros adotados nos experimentos do AG

\begin{tabular}{|c|r|}
\hline Parâmetro & \multicolumn{1}{|c|}{ Valor } \\
\hline Número de gerações & 100 \\
\hline Tamanho da população & 100 \\
\hline Probabilidade de cruzamento & $0.9(90 \%)$ \\
\hline Probabilidade de mutação & $0.1(10 \%)$ \\
\hline Quantidade de alunos por equipe & 3 \\
\hline
\end{tabular}

\subsection{Resultados e Validação da Proposta}

As Tabelas 2, 3 e 4 apresentam os resultados obtidos pelas estratégias por escolha mútua, escolha aleatória e o AG proposto, respectivamente. Para a escolha aleatória e o AG 
VIII Congresso Brasileiro de Informática na Educação (CBIE 2019)

Anais do XXX Simpósio Brasileiro de Informática na Educação (SBIE 2019)

estão dispostos o maior e menor valor de heterogeneidade atingidos, bem como a média, considerando 30 execuções de cada abordagem em cada turma. A configuração de equipes da estratégia por escolha mútua foi obtida mediante solicitação aos alunos para realizarem os agrupamentos levando em conta a afinidade entre os mesmos, logo, as equipes não sofrem modificações e o resultado do DB é único para cada turma.

Em uma análise geral, os resultados evidenciam que a estratégia por escolha mútua, abordagem usualmente empregada para a formação de equipes em prol da aprendizagem, trata-se da alternativa de pior desempenho, tendo seus resultados inferiores e superados até mesmo por uma abordagem totalmente aleatória de escolha dos alunos. Por outro lado, o AG proposto apresenta-se como o método mais propício à formação de equipes heterogêneas, alcançando distribuição de equipes que maximizam a heterogeneidade quando comparadas com as abordagens tradicionais, ainda que o método aleatório encontre soluções de valor igual ou superior ao AG em certas ocasiões.

Tabela 2. Resultados da estratégia por escolha mútua

\begin{tabular}{|r|r|}
\hline Turma & Resultado \\
\hline 2009 & 1.58 \\
\hline 2010 & 2.10 \\
\hline 2011 & 1.31 \\
\hline 2012 & 2.00 \\
\hline 2013 & 0.93 \\
\hline 2014 & 1.57 \\
\hline 2015 & 1.06 \\
\hline
\end{tabular}

Tabela 3. Resultados da estratégia por escolha aleatória

\begin{tabular}{|r|r|r|r|}
\cline { 2 - 4 } \multicolumn{1}{c|}{} & \multicolumn{3}{c|}{ Resultado } \\
\hline Turma & Maior & Menor & Média \\
\hline 2009 & 2.15 & 1.78 & 1.99 \\
\hline 2010 & 2.71 & 2.23 & 2.43 \\
\hline 2011 & 2.71 & 2.16 & 2.46 \\
\hline 2012 & 5.13 & 3.81 & 4.27 \\
\hline 2013 & 1.99 & 1.55 & 1.89 \\
\hline 2014 & 2.80 & 2.29 & 2.46 \\
\hline 2015 & 2.47 & 2.15 & 2.29 \\
\hline
\end{tabular}

Tabela 4. Resultados do Algoritmo Genético

\begin{tabular}{|r|r|r|r|}
\cline { 2 - 4 } \multicolumn{1}{c|}{} & \multicolumn{3}{c|}{ Resultado } \\
\hline Turma & Maior & Menor & Média \\
\hline 2009 & 2.26 & 1.93 & 2.05 \\
\hline 2010 & 2.92 & 2.38 & 2.64 \\
\hline 2011 & 2.75 & 2.36 & 2.53 \\
\hline 2012 & 5.03 & 4.00 & 4.52 \\
\hline 2013 & 1.99 & 1.59 & 1.97 \\
\hline 2014 & 2.90 & 2.35 & 2.60 \\
\hline 2015 & 2.62 & 2.21 & 2.39 \\
\hline
\end{tabular}

Todavia, ainda que os resultados do AG proposto tenham se sobressaído às demais abordagens, tal aspecto não garante que, de fato, o mesmo possua efeito significativo 
VIII Congresso Brasileiro de Informática na Educação (CBIE 2019)

Anais do XXX Simpósio Brasileiro de Informática na Educação (SBIE 2019)

na heterogeneidade das equipes em comparação à escolha mútua e aleatória. Para isso, tornou-se necessária a utilização de testes estatísticos a fim de analisar a influência do AG para com a formação de equipes heterogêneas, assim verificando se esta abordagem produz impacto significativo na maximização da heterogeneidade das equipes em turmas universitárias.

Dessa forma, a Tabela 5 retrata o resultado da Análise de Variância (ANOVA) acerca dos resultados das estratégias. Conforme observado, o valor de $F_{\text {calc }}$ é superior ao $F_{t a b}$ e, consequentemente, é válido afirmar que as estratégias possuem diferença estatística em seus resultados. Portanto, a hipótese que o AG proposto é superior às estratégias tradicionais é reafirmada em termos estatísticos, apresentando-se como um método eficaz para a formação de equipes de modo a garantir a heterogeneidade nos agrupamentos.

Tabela 5. Resultados do teste ANOVA

\begin{tabular}{|c|c|c|c|c|c|c|}
\hline Alfa & 0,05 & & & & & \\
\hline Grupos & Contagem & Soma & Média & Variância & & \\
\hline Escolha mútua & 7 & 10,55 & 1,51 & 0,20 & & \\
\hline Escolha aleatória & 7 & 17,79 & 2,54 & 0,63 & & \\
\hline Algoritmo Genético & 7 & 18,7 & 2,67 & 0,73 & & \\
\hline $\begin{array}{l}\text { Origem de } \\
\text { variações }\end{array}$ & $\begin{array}{c}\text { Soma de } \\
\text { quadrados }\end{array}$ & $\begin{array}{l}\text { Graus de } \\
\text { liberdade }\end{array}$ & $\begin{array}{l}\text { Quadrados } \\
\text { médios }\end{array}$ & $F_{\text {calc }}$ & Valor $p$ & $F_{t a b}$ \\
\hline Entre grupos & 5,70 & 2 & 2,85 & 5,47 & 0,01 & 3,55 \\
\hline Dentro dos grupos & 9,38 & 18 & 0,52 & & & \\
\hline Total & 15,08 & 20 & & & & \\
\hline
\end{tabular}

\section{Conclusões e Trabalhos Futuros}

Os resultados deste estudo demonstraram que os métodos comumente empregados para a realização de agrupamentos de alunos no âmbito acadêmico não favorecem, em grande parte dos casos, a heterogeneidade das equipes. O agrupamento por afinidade preserva equipes de caráter homogêneo e impossibilita a obtenção de uma configuração que permita diversificar os alunos. Por outro lado, a estratégia aleatória é incapaz de abranger o conjunto de combinações possíveis e encontrar uma configuração ótima de equipes que maximize a heterogeneidade. Um esquema baseado em algoritmos genéticos é proposto e validado através de experimentos com dados reais e testes estatísticos, destacando-se como o método mais eficaz para a garantia de dissimilaridade entre alunos em equipes.

Como trabalhos futuros, destacam-se a análise de diferentes parâmetros no AG que possam proporcionar soluções de maior qualidade, a consideração de outros atributos para a realização dos agrupamentos, como dados socioeconômicos e perfil emocional, podendo garantir uma maior heterogeneidade nas equipes, além do estudo de outras abordagens meta-heurísticas que possam contribuir significativamente para o problema abordado.

\section{Referências}

Balieiro, A., Melo, I., Araújo, D., Neto, S., Galdino, E., and Gomes, A. (2017). Uma abordagem baseada em algoritmo genético para formação de grupos de estudos em ambientes virtuais de aprendizagem. In Simpósio Brasileiro de Informática na EducaçãoSBIE, pages 1287-1296. 
VIII Congresso Brasileiro de Informática na Educação (CBIE 2019)

Anais do XXX Simpósio Brasileiro de Informática na Educação (SBIE 2019)

Bandyopadhyay, S. and Maulik, U. (2002). Genetic clustering for automatic evolution of clusters and application to image classification. Pattern Recognition, 35(6):1197 1208.

Blum, C. and Roli, A. (2003). Metaheuristics in combinatorial optimization: Overview and conceptual comparison. ACM computing surveys (CSUR), 35(3):268-308.

Cen, L., Ruta, D., Powell, L., and Ng, J. (2015). Interaction driven composition of student groups for optimal groupwork learning performance. In Proceedings of the 2015 IEEE Frontiers in Education Conference (FIE), pages 1-6, Washington, DC, USA. IEEE Computer Society.

Chen, L. and Yang, Q. H. (2014). A group division method based on collaborative learning elements. In The 26th Chinese Control and Decision Conference (2014 CCDC), pages 1701-1705.

Coley, D. A. (1999). An introduction to genetic algorithms for scientists and engineers. World Scientific Publishing Co Inc.

Cooke, N. J., Gorman, J. C., Myers, C. W., and Duran, J. L. (2013). Interactive team cognition. Cognitive Science, 37(2):255-285.

Holland, J. H. (1992). Adaptation in Natural and Artificial Systems: An Introductory Analysis with Applications to Biology, Control and Artificial Intelligence. MIT Press, Cambridge, MA, USA.

Larose, D. T. (2014). Discovering knowledge in data: an introduction to data mining. John Wiley \& Sons.

Lin, Y. S., Chang, Y. C., and Chu, C. P. (2016). Novel approach to facilitating tradeoff multi-objective grouping optimization. IEEE Transactions on Learning Technologies, 9(2):107-119.

Macintyre, H. and Ireson, J. (2002). Within-class ability grouping: Placement of pupils in groups and self-concept. British Educational Research Journal, 28(2):249-263.

Maldonado Pérez, M. (2007). El trabajo colaborativo en el aula universitaria. Laurus, 13(23).

Mitchell, M. (1998). An introduction to genetic algorithms. MIT press.

Moreno, J., Ovalle, D. A., and Vicari, R. M. (2012). A genetic algorithm approach for group formation in collaborative learning considering multiple student characteristics. Computers \& Education, 58(1):560 - 569.

Silva, H. M., Silva, C. A., and Gorgônio, F. L. (2012). A self-organizing map based strategy for heterogeneous teaming. In Johnsson, M., editor, Applications of SelfOrganizing Maps, chapter 05. InTech, Rijeka.

Torres, T. Z. and Amaral, S. F. d. (2011). Aprendizagem colaborativa e web 2.0: proposta de modelo de organização de conteúdos interativos. Educação Temática Digital, 12:49.

Zheng, Y., Liu, Y., Lu, W., and Li, C. (2016). A hybrid pso-ga method for composing heterogeneous groups in collaborative learning. In 2016 11th International Conference on Computer Science Education (ICCSE), pages 160-164. 